\title{
Prosthodontic maintenance and peri-implant tissue conditions for telescopic attachment-retained mandibular implant overdenture: Systematic review and meta-analysis of randomized clinical trials
}

\author{
Ahmed Mohamed Keshk ${ }^{1}$, Ahmed Yaseen Alqutaibi ${ }^{2}$, Radhwan S. Algabri ${ }^{1}$, \\ Mostafa S. Swedan ${ }^{1}$, Amal Kaddah ${ }^{1}$
}

Correspondence: Dr. Ahmed Mohamed Keshk Email:dr_magic_pearl@hotmail.com
'Department of Prosthodontics, Faculty of Oral and Dental Medicine, Cairo University, Cairo, Egypt, ${ }^{2}$ Department of Prosthodontics, Faculty of Oral and Dental Medicine, Ibb University, Ibb, Yemen,

\section{ABSTRACT}

The mandibular implant-retained overdentures (MIRO) are a highly successful prosthetic treatment option. However, an argument still present regarding its design and type of attachment system. This systematic review and meta-analysis aimed to perform a qualitative and quantitative analysis of the scientific literature regarding the telescopic attachments versus other attachment systems retaining mandibular implant overdentures. Manual and electronic database (PubMed and Cochrane) searches were performed to identify randomized controlled trials (RCTs) comparing telescopic versus other attachment systems. Independently, two investigators extracted the trials' data. The Cochrane tool was used for assessing the quality of included studies. Meta-analyses were performed for the included RCTs and reported the same outcome measures. Nine RCTs were identified. Three RCTs (corresponding to four publications) were included in the study. The other five trials were excluded from the study. The meta-analysis revealed no difference between telescopic crowns and ball attachment retaining mandibular implant overdenture as regards prosthodontic maintenance. Regarding peri-implant conditions, ball-retained mandibular overdenture showed statistically significant more probing depth around implants records in ball-retained overdenture when compared to the telescopic group. However, there are no statistically significant differences between two interventions in regard to marginal bone loss, bleeding index, gingival index, and plaque index. In conclusions, no significant differences in prosthodontic maintenance and peri-implant condition between telescopic attachments and ball attachments retaining MIRO. However, this should be considered with caution because of a limited number of included studies.

Key words: Ball attachment, bar attachment, implant overdenture, systematic review, telescopic attachment

\section{INTRODUCTION}

Usually, edentulous patients are complaining of difficulties during chewing and speaking leading to a decline in their quality of life. Especially in the mandibular arch, the space available to the prosthesis

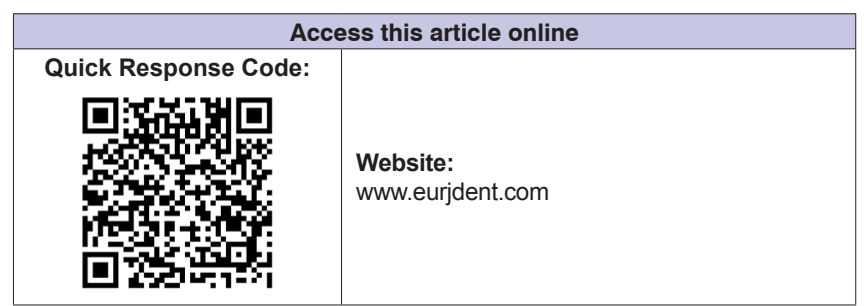

and its stability are reduced due to the presence of the tongue. The placement of two or more dental implants

This is an open access article distributed under the terms of the Creative Commons Attribution-NonCommercial-ShareAlike 3.0 License, which allows others to remix, tweak, and build upon the work non-commercially, as long as the author is credited and the new creations are licensed under the identical terms.

For reprints contact: reprints@medknow.com

How to cite this article: Keshk AM, Alqutaibi AY, Algabri RS,
Swedan MS, Kaddah A. Prosthodontic maintenance and peri-implant
tissue conditions for telescopic attachment-retained mandibular implant
overdenture: Systematic review and meta-analysis of randomized clini-
cal trials. Eur J Dent 2017;11:559-68.
DOI: $10.4103 /$ ejd.ejd_23_17


in the anterior mandible provides additional means of retention to stabilize mandibular overdentures and affords a safe and long-term clinical success. ${ }^{[1-3]}$

Mandibular implant-retained overdentures (MIRO) present a reliable and simple solution to enhance denture retention and stability. The retention and stability characteristics are provided mainly by implants through attachments. Hence, various types of attachment systems have been proposed for connecting implant-retained mandibular overdentures to the underlying implants. ${ }^{[4]}$

Splinted attachments as bar attachments is a popular choice because of its load sharing but requires sufficient interarch space; ${ }^{[4]}$ it may cause mucosal hyperplasia underneath the bar if insufficient relief is present, and contraindicated to be used with a $\mathrm{V}$-shaped ridge to avoid encroaching on the tongue space. ${ }^{[5]}$

Non-splinted attachments as telescopic and ball attachments. ${ }^{[6,7]}$ Ball attachments are susceptible to wear and technique sensitive as they require parallel implants placement. ${ }^{[8]}$ Telescopic attachments have excellent retention due to frictional fit between primary and secondary copings. The circumferential relation between telescopic attachment and the abutment allows better distribution of forces, results in transferring the occlusal load more axially leads to reducing the rotational torque on the abutment. ${ }^{[9,10]}$ However, they require enough inter-arch space to be occupied. If there is no sufficient inter-arch space, telescopic attachment is not recommended to be used. $^{[11]}$

To assess a MIRO, the implant survival rate and the complication rate are the most important factors. ${ }^{[12]}$ To determine an implant prosthesis survival, it is better to mention "time to retreatment" ${ }^{[13]}$ which is the time needed to perform any interference by the clinician to manage any prosthetic complications during the maintenance period. ${ }^{[14]}$

Implant overdentures complications may be biologic and technical complications. Biologic complications are any disturbances in implant function that affect the supporting peri-implant tissues in terms of early or late implant failures, and adverse reactions in the periimplant hard and soft tissues. Technical complications are any mechanical damage of the implant, implant components, and suprastructures. ${ }^{[15]}$ Prosthetic complications are the need of the final prosthesis after the insertion to be relined or repaired although it affects or not affects implant. ${ }^{[16]}$
In this review, the question is, Could the telescopic attachments in completely edentulous patients needing dental implant rehabilitation better than other attachment systems regarding implant survival, complications, and peri-implant tissue condition?

\section{MATERIALS AND METHODS}

\section{Protocol registration}

A prior protocol was made and registered at PROSPERO with registration NO: CRD42017054762.

\section{The review structure}

The "Preferred Reporting Items for Systematic Reviews and Meta-Analyses" was followed. ${ }^{[17]}$

\section{Eligibility criteria}

According to the PICOS format provided by the Center for Evidence-Based Medicine, the focused question was formulated and served as a basis for the systematic literature search. ${ }^{[18]}$

- Population/problem: Completely edentulous patients with a MIRO

- Interventions: Telescopic crown attachments retaining MIRO

- Comparators: Other attachment systems (Ball, Bar, and Locator) retaining MIRO

- Outcome: Any outcome not predetermined or included in the search strategy

- Study design: Randomized-controlled trials (RCTs).

\section{Exclusion criteria}

Case report, case series studies, retrospective studies, cohort studies, animal studies, in vitro studies, or non-RCTs were not included. And any observation period of leass than 3 years.

\section{Search strategy}

The search was performed by two reviewers independently. Combinations of controlled terms $(\mathrm{MeSH})$ and keywords were used whenever possible [Table 1]. A comprehensive electronic search was done in both PubMed and the Cochrane Central Register of controlled trials databases with language restriction to English only and without time restrictions. Furthermore, a manual search was done in the related journals, including; the Journal of Prosthodontics, the International Journal of Prosthodontics, Journal of Advanced Prosthodontics, the Journal of Prosthodontic Research, Journal of oral rehabilitation, Journal of Dental Research, and Journal of Oral and Maxillofacial surgery. Moreover, online databases providing information about clinical trials 


Table 1: The search terms used for the search in
electronic databases
(edentulous jaw) OR (edentulous mouth) OR (edentulous ridge)
OR (edentulous arch) OR (edentulous mandible) OR (completely
edentulous patient) OR (totally edentulous patient) OR (mandibular
prosthesis) OR (mandibular prostheses) OR (mandibular
overdenture) OR (mandibular implant retained overdenture)
OR (mandibular implant assisted overdenture) OR (mandibular
implant supported overdenture) OR (implant overlay) OR (implant
prosthesis) AND (telescopic attachment) (OR telescopic
crown) OR (telescopic overdenture) OR (telescopic prosthesis)
OR (telescopic prostheses) OR (double crown) OR (double crowns)
OR (double-crown) OR (conus attachment) OR (conical crown)
OR (conical attachment) AND (bar attachment) OR (attachment
bar) OR (bar overdenture) OR (bar overdentures) OR (bar retained
implant overdenture) OR (ball attachment) OR (ball overdenture)
OR (ball retained implant overdenture) OR (ball and socket)
OR (locator attachment) OR (locator overdentures)

in progress were checked such as www.clinicaltrials. gov, www.centerwatch.com/clinicaltrials, and www. clinicalconnection.com. The last performed search was on December 5, 2016.

\section{Study selection}

Study selection and data extraction were performed independently by two reviewers and any disagreement was solved by discussion. If not, a third reviewer was consulted.

\section{Data extraction}

Two reviewers performed the data extraction independently and were reciprocally blinded to the extraction each other. The following information was extracted: author, country, follow-up year, age of the patient, gender, implant system, number of participants, the total number of implant placed, interventions, attachment system, participants per group, participant analyzed, implant per participant, implant survival rate, prosthetic maintenance, and peri-implant condition.

\section{The quality assessment (risk of bias)}

The risk of bias assessment of the included trials was done by two reviewers independently using the Cochrane collaboration's tool, ${ }^{[19]}$ six specific domains titled sequence generation, allocation concealment, blinding, incomplete outcome data, selective outcome reporting, and other bias. An RCT was assigned (Low risk of bias) if all domains were at low risk of bias, (Unclear risk of bias) if there was unclear risk of bias of at least one domain, and (High risk of bias) if at least one domain was scored as being at a high risk of bias. In the case of disagreement, discussion between the two reviewers reveals final decisions.

\section{Statistical analyses}

Measures of treatment effect

For dichotomous outcomes, the effect of an intervention was expressed as risk differences (RDs) together with 95\% confidence intervals (CIs). However, for continuous outcomes, mean differences (MDs) and standard deviations were used to summarize the data for each group with 95\% CIs.

\section{Unit of analysis issues}

The statistical unit was the patient.

Missing data

If there is any relevant missing information in the included articles, the corresponding authors of these articles were contacted by E-mail. In the situations of no responses, reminder E-mails were sent.

\section{Data synthesis}

All statistical tests were performed using the Review Manager (RevMan) software release version 5.3. ${ }^{[20]}$ RevMan is The Cochrane Collaboration's software for preparing and maintaining Cochrane reviews. Meta-analyses were done for studies reported the same outcomes. Risk differences (RDs) for prosthodontic maintenance and MDs for peri-implant tissue were calculated and compared between the two studied interventions (telescopic crown versus ball attachment retaining mandibular implant overdenture). CIs were set at $95 \%$. Weighted means across the studies were calculated using a fixed-effects model. A random-effects model was used to assess the significance of treatment effects.

\section{Heterogeneity assessment}

Cochran's test for heterogeneity was used to assess any variations significance in the estimates of the treatment effects of the different trials, heterogeneity would be considered significant if $P<0.1$. Heterogeneity between the studies was assessed using the $I^{2}$-statistic, which describes the variation percentage due to heterogeneity rather than chance. ${ }^{[21]} I^{2}$ over $50 \%$ was considered as moderate to high heterogeneity.

\section{Reporting biases assessment}

If there had been sufficient numbers of trials $(>10)$ in any meta-analyses, publication bias would have been assessed according to funnel plot asymmetry. If asymmetry was identified, we would have examined possible causes. 


\section{RESULTS}

The electronic search yielded a total of 54 articles (PubMed $=36$ and The Cochrane Library $=13) .5$ records identified from other sources. 25 potentially relevant articles were selected after screening with title and abstract and removing duplicates. After the initial screening, nine potentially eligible RCTs, ${ }^{[12,22-29]}$ four publications ${ }^{[22,27-29]}$ were included and five publications ${ }^{[12,23-26]}$ were excluded. Reasons for exclusion were as follows: Three were nonrandomized clinical trials. ${ }^{[12,23,25]}$ One was of unclear data. ${ }^{[26]}$ One had a period of follow-up $<1$ year (3 months). ${ }^{[24]}$

Only two trials were subsequently analyzed in this systematic review [Figure 1]. Details of all included studies are summarized in Tables 2 and 3.

\section{Characteristics of included studies}

The included publications were published within 10 years (2006-2016). All of them were RCTs that examined edentulous mandibular arches. Their observation periods were 3 years, but one of them ${ }^{[27]}$ has a follow-up period of 5 years.

One of the include trials is a 3-year follow-up study ${ }^{[29]}$ which is a part of extended another trial of 5-year follow-up; $;^{[27]}$ therefore, both studies are included in one study.

One trial ${ }^{[22]}$ conducted in Germany, the other three trial ${ }^{[27-29]}$ in Austria. All trials conducted in university dental clinics and their study protocols were approved by the Local Ethics Committee of their universities.

Three of the included trials compared the effect of telescopic versus ball attachments ${ }^{[22,27,29]}$ and

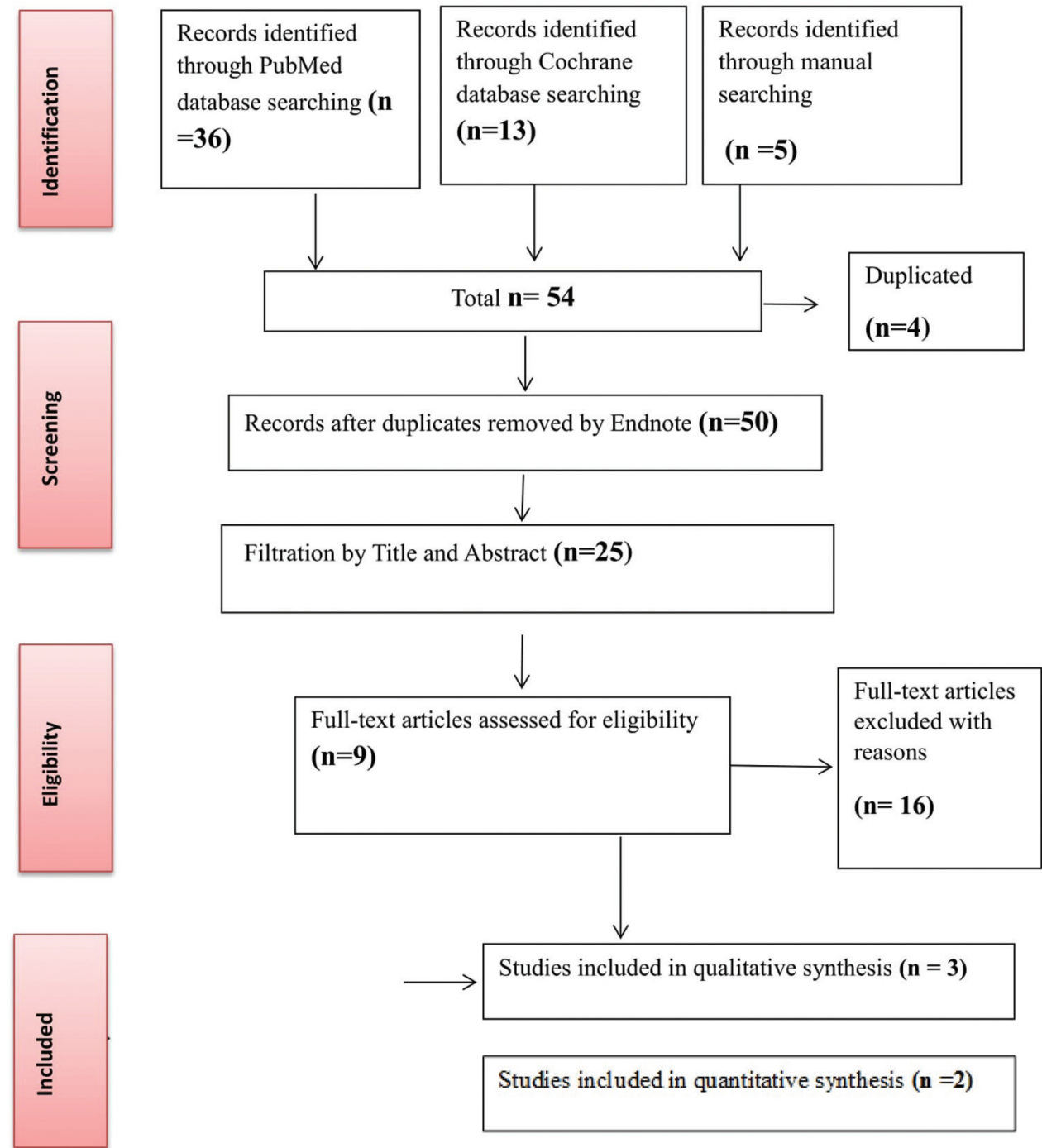

Figure 1: Preferred Reporting Items for Systematic Reviews and Meta-Analyses flow chart 
Keshk, et al.: Telescopic attachment-retained mandibular implant overdenture: Systematic review
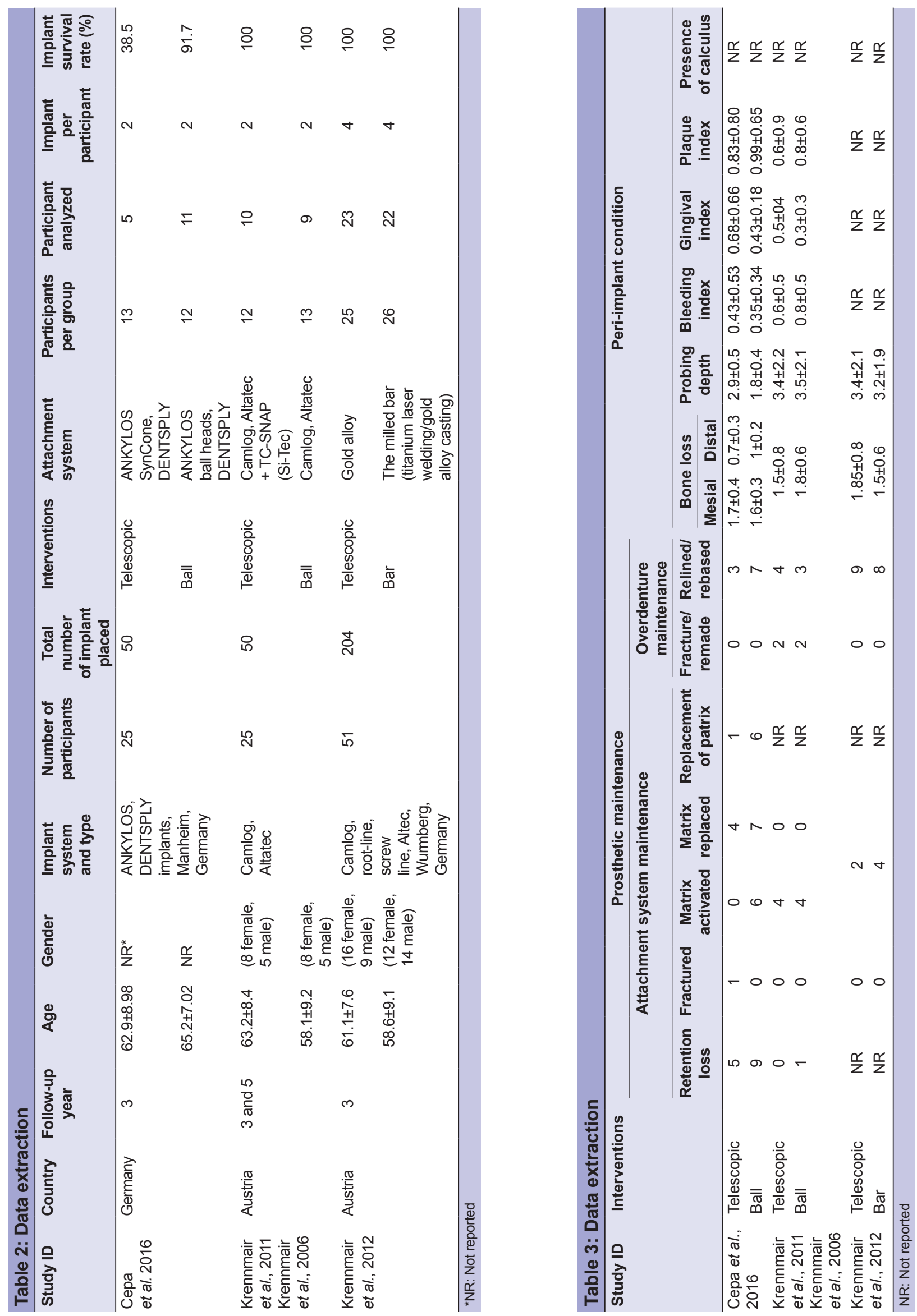
one compared the effect of telescopic versus bar attachments ${ }^{[28]}$ retaining mandibular implant overdentures.

The same participants' number was (25 patients) in three trials that compare between the telescopic and ball attachments retaining mandibular implant overdentures. ${ }^{[2,27,29]}$ One trial had 51 participants which compared the effect of telescopic versus bar attachments ${ }^{[28]}$ retaining mandibular implant overdenture.

The inclusion or exclusion criteria of patients were reported clearly in one trial. ${ }^{[22]}$ However, regarding the other 3 trials, they are not mentioned ${ }^{[27-29]}$ in all included trials, each patient was given a detailed prescription of the planned procedures and signed a written informed consent before participation.

One hundred and twenty-six patients received 354 implants. All implants were titanium implants, had various types and surface modifications and with different lengths and diameters. Implant numbers per patient varied between $2 \mathrm{implants}$ in the mandible ${ }^{[22,27,29]}$ and 4 implants in the mandible. ${ }^{[28]}$ The mandibular interforaminal area was the implant positioning preferred area. A two-stage surgical procedure and conventional loading protocol were followed.

The outcomes were reported as follows:

- Implant survival rate (reported in all trials)

- Prosthodontic maintenance, which subdivided into two categories: (1) attachment system maintenance in terms of retention loss, fracture, matrix activated, matrix replaced, and replacement of patrix. (2) The overdenture maintenance in terms of overdenture fractured/remade and overdenture relining/ rebased

- Peri-implant tissue condition evaluation in terms of plaque indices, bleeding indices, gingival indices, and probing depth. A radiographic evaluation was done to measure the marginal bone level around implants.

\section{Quality assessment}

The risk of bias assessment of the included trials is summarized in Table 4. Each trial was assessed to be at low, unclear, or high risk of bias. Two of three included RCTs were assessed to be at high risk and one at unclear risk of bias.

\section{Effects of interventions}

After 3-year follow-up, Cepa et al. ${ }^{[22]}$ evaluated 25 patients with completely edentulous mandibular arches for implant survival, peri-implant tissue parameters, and patient satisfaction regarding two different attachment systems (ball and telescopic) retaining implant mandibular overdentures. Randomly, twelve patients have received ball attachments, and other thirteen patients received the prefabricated telescopic attachment. All follow-ups were done and documented annually up to 3 years.

The results showed 100\% implant survival rate. No significant differences in the peri-implant tissue evaluation. About $64 \%$ of patients that received ball attachments were satisfied, but $100 \%$ patients that received telescopic attachments were satisfied. The latter only respecting five of initially 13 patients. In addition, Cepa et al. ${ }^{[22]}$ concluded that the ball attachments group required intensive prosthetic maintenance.

Krennmair et al. ${ }^{[27,29]}$ observed implant success, peri-implant conditions, prosthodontic maintenance, and patient satisfaction annually during a 5-year follow-up period by comparing ball and telescopic attachments retaining mandibular implant overdentures. Krennmair et al. published two articles, one during a 3-year period ${ }^{[29]}$ and the other after a 5-year period. ${ }^{[27]}$ Twenty-five patients were randomly distributed into; 13 patients received ball attachments and 12 patients received telescopic crowns.

The results revealed that peri-implant tissue conditions, implant survival rate, and subjective patient satisfaction scores did not show the difference between the ball and telescopic attachments. After 5-year follow-up, the prosthodontic maintenance was more significant in the ball group (87 interventions, $61.1 \%)$ than in the telescopic attachments group (53 interventions, $37.9 \% ; P<0.01$ ). In the second and third years, differences in prosthodontic maintenance efforts were most significant $(P<0.05)$ but both were

\section{Table 4: The risk of bias assessment}

\begin{tabular}{|c|c|c|c|c|c|c|}
\hline Study ID & $\begin{array}{l}\text { Random } \\
\text { sequence }\end{array}$ & Allocation concealment & Blinding & Incomplete outcome data & Selective reporting & Others \\
\hline Cepa et al. ${ }^{[22]}$ & Low risk & Low risk & Unclear & Low risk & Low risk & Low risk \\
\hline Krennmair et al. ${ }^{[27,29]}$ & Unclear & High risk & Unclear & Low risk & Low risk & Low risk \\
\hline Krennmair et al. ${ }^{[28]}$ & Unclear & High risk & Unclear & Low risk & Low risk & Low risk \\
\hline
\end{tabular}


similar at the end of the study for both attachment systems.

Krennmair et al. ${ }^{[2]}$ concluded that $100 \%$ implant survival rate, good peri-implant tissue conditions, and general patient satisfaction were scored. Although the higher prosthetic maintenance incidence of the ball attachments group than of the telescopic attachments, similar frequencies of maintenance efforts may be anticipated for both retention systems over a 5-year period.

During a 3-year follow-up period, Krennmair et al..$^{[28]}$ evaluated 45 patients (dropout rate: $45 / 51=11.8 \%$ ) who received four mandibular interforaminal implants in the edentulous mandible and complete maxillary dentures. Randomly, 23 patients were received milled bars and 22 patients received telescopic attachments.

The results showed high implant survival rate $(100 \%)$. Peri-implant marginal bone resorption, pocket depth as well as bleeding index and gingival index did not differ for both retention systems. However, annually higher values for plaque index (NS) and calculus index $(P<0.035)$ were noticed for the bar than for the telescopic attachments.

Prevalence of prosthodontic maintenance did not differ between both retention modalities. However, prosthodontic adaption for handling mechanism showed benefits for the bar retention.

Krennmair et al. ${ }^{[28]}$ concluded drawbacks such as higher plaque/calculus for bar retention and less favorable handling properties (output) for telescopic crown attachment leave the selection decision on the clinician.

\section{Meta-analysis}

A meta-analysis was performed for the studies having same comparison groups and same outcomes.

\section{Prosthetic maintenance}

The meta-analyses of two trials ${ }^{[22,29]}$ regarding the need for prosthetic maintenance comparing telescopic and ball-retained mandibular overdenture showed no differences between two interventions in regard to matrix activation, matrix replacement, patrix replacement, overdenture relining, and overdenture remake [Figure 2].

\section{Peri-implant conditions}

The meta-analyses of two trials ${ }^{[22,29]}$ regarding peri-implant conditions comparing telescopic and ball-retained mandibular overdenture showed statistically significant more probing depth around implants records in ball-retained overdenture when compared to telescopic group $\left(I^{2}=47 \%, P=0.00001\right.$; MD: $1.1,95 \%$ CI: 0.52, 1.48). However, there are no statistically significant differences between two interventions in regard to marginal bone loss, bleeding index, gingival index, and plaque index [Figure 3].

\section{DISCUSSION}

The MIRO gave the best results compared to the conventional removable prostheses resulting in improved quality of life, the masticatory efficiency, and therefore, the nutritional condition and patient's health. ${ }^{[30]}$

The MIRO represents a first choice option, especially when there is a need to anchor the mandibular conventional denture. It is important to remember that dental implants, although they afford the overdenture with enhanced retention and support, differ significantly from the natural teeth. The most important difference from the biomechanical point of view is the absence of the periodontal ligament (PDL), which performs the amortization functions of occlusal loads, the proprioceptive sensitivity, and promotes bone regeneration activities. ${ }^{[31]}$

Under loading forces over the natural teeth, the PDL involved first followed by the alveolar bone. However, dental implant, due to the absence of the PDL, has a linear model of the deflection force that depends on the elastic deformation of the alveolar bone. ${ }^{[32]}$

This review delivers meta-analyses of the RCTs that is considered as the highest level of confirmatory scientific evidence today. ${ }^{[33]}$ In terms of internal validity, RCTs represent the most scientifically rigorous study designs, as they are best able to control bias and serve as a gold standard of study designs for evaluating treatment efficacy. ${ }^{[34]}$

The meta-analysis of the two included RCTs ${ }^{[22,29]}$ reveals that when comparing telescopic and ball-retained mandibular overdenture, there are no differences between two interventions regarding the need for prosthetic maintenance. This is in agreement with MacEntee et al..$^{[35]}$ and Watson et al. ${ }^{[36]}$ who record no differences regarding postinsertion maintenance between interventions of the attachment systems that being compared. 
Keshk, et al.: Telescopic attachment-retained mandibular implant overdenture: Systematic review

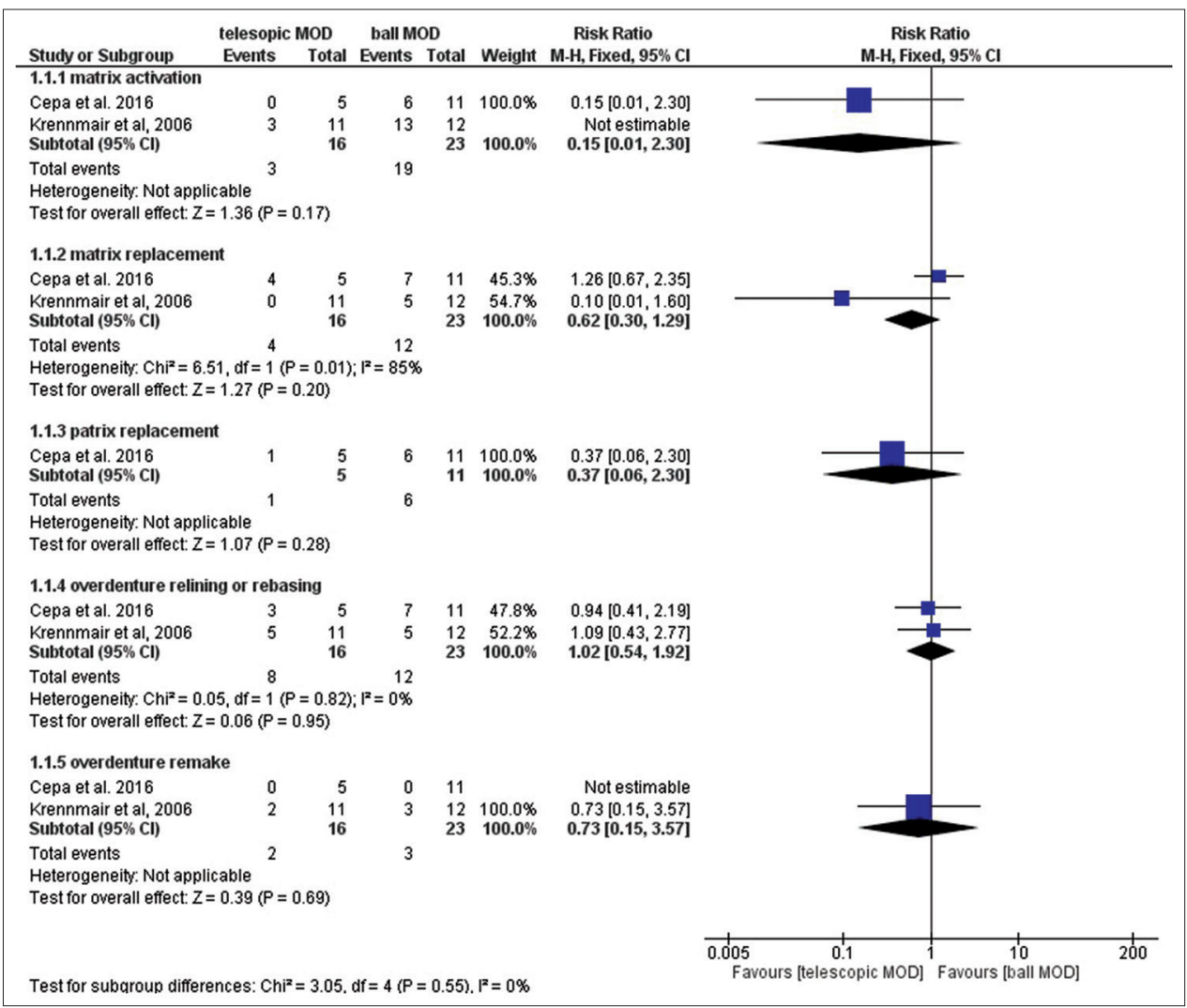

Figure 2: Forest plot telescopic versus ball implants-retained mandibular overdenture: prosthodontic maintenance

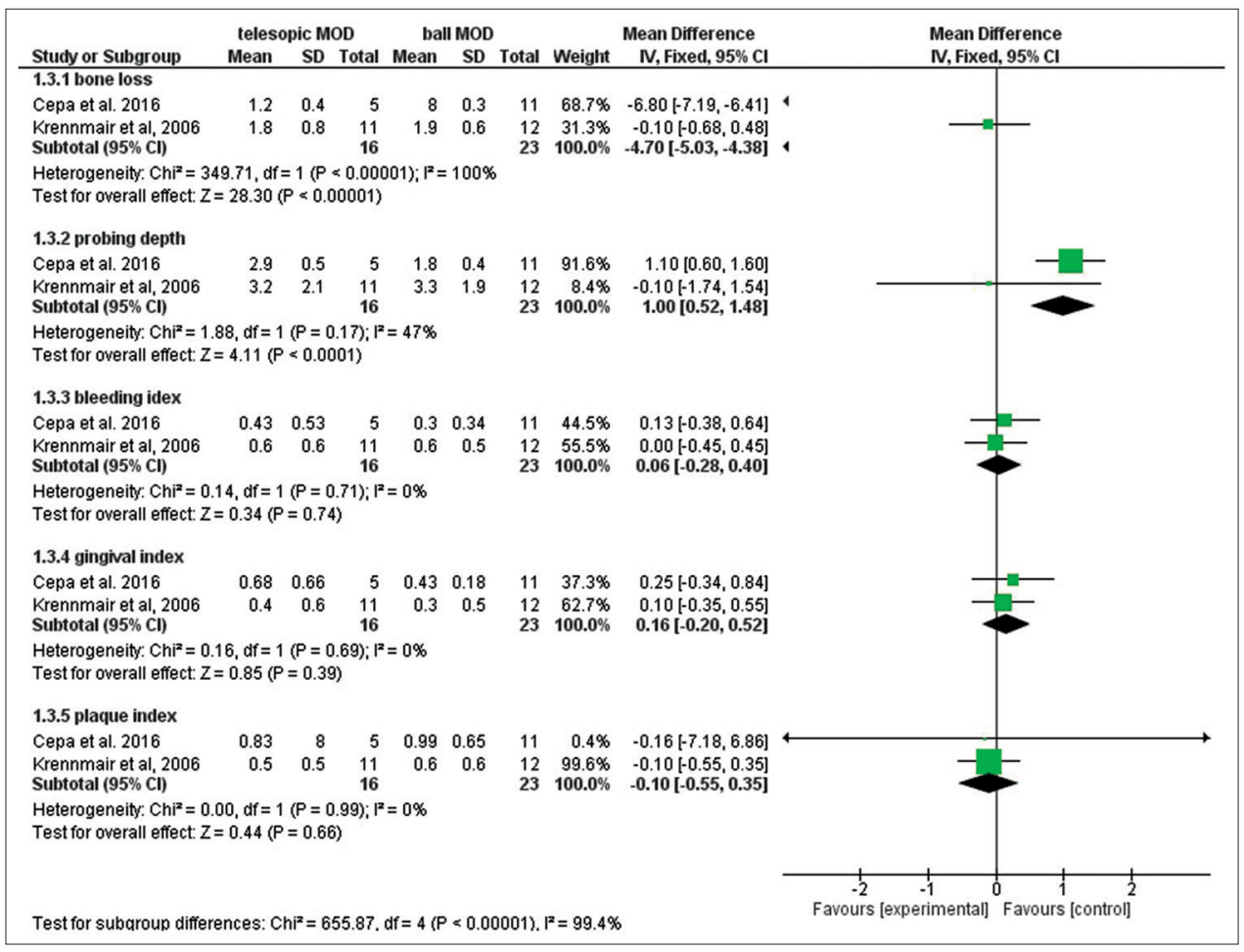

Figure 3: Forest plot telescopic versus ball implants-retained mandibular overdenture: peri-implant condition

Karabuda et al. ${ }^{[37]}$ found similar results. They compared overdentures with bar and ball abutment on two to four implants in 26 patients. The treatment success with both techniques was also compared with our 
meta-analysis as they reported a total of 20 prosthetic complications were recorded in both groups. No differences in prosthetic complications were observed for two attachment systems.

Regarding peri-implant tissue conditions, when comparing the telescopic and ball retained mandibular overdenture the results showed statistically significant more probing depth around implants. This may be explained by bone remodeling and consolidation of biological width after implant placement. ${ }^{[38,39]}$

However, the meta-analysis showed no statistically significant differences between two interventions in regard to marginal bone loss, bleeding index, gingival index, and plaque index. Naert et al. ${ }^{[40]}$ studied the influence of splinted and unsplinted oral implants retaining mandibular overdentures. Over 10 years, no implants failed. Mean plaque index, bleeding index, change in attachment level, periotest values, and marginal bone level at the end of the follow-up period were not significantly different among the groups. Periotest values and marginal bone level at the end of the follow-up period were not significantly different among the groups.

\section{CONCLUSION}

The meta-analysis revealed no significant difference regarding peri-implant tissue condition and prosthodontic maintenance when comparing telescopic attachments with ball attachments. However, this should be interpreted with caution because limited number included studies. More well-designed RCTs are highly recommended to evaluate the effectiveness of telescopic versus other attachment systems retaining mandibular implant overdentures.

\section{Financial support and sponsorship Nil.}

\section{Conflicts of interest}

There are no conflicts of interest.

\section{REFERENCES}

1. Michailidis N, Karabinas G, Tsouknidas A, Maliaris G, Tsipas D, Koidis P. A FEM based endosteal implant simulation to determine the effect of peri-implant bone resorption on stress induced implant failure. Biomed Mater Eng 2013;23:317-27.

2. Cicciù M, Beretta M, Risitano G, Maiorana C. Cemented-retained vs. screw-retained implant restorations: An investigation on 1939 dental implants. Minerva Stomatol 2008;57:167-79.

3. Cicciù M, Cervino G, Bramanti E, Lauritano F, Lo Gudice G, Scappaticci L, et al. FEM analysis of mandibular prosthetic overdenture supported by dental implants: Evaluation of different retention methods. Comput Math Methods Med 2015;2015:943839.

4. Kitagawa T, Tanimoto Y, Odaki M, Nemoto K, Aida M. Influence of implant/abutment joint designs on abutment screw loosening in a dental implant system. J Biomed Mater Res B Appl Biomater 2005;75:457-63.

5. Karabuda C, Tosun T, Ermis E, Ozdemir T. Comparison of 2 retentive systems for implant-supported overdentures: Soft tissue management and evaluation of patient satisfaction. J Periodontol 2002;73:1067-70.

6. Cune M, van Kampen F, van der Bilt A, Bosman F. Patient satisfaction and preference with magnet, bar-clip, and ball-socket retained mandibular implant overdentures: A cross-over clinical trial. Int J Prosthodont 2005;18:99-105.

7. van Kampen F, Cune M, van der Bilt A, Bosman F. Retention and postinsertion maintenance of bar-clip, ball and magnet attachments in mandibular implant overdenture treatment: An in vivo comparison after 3 months of function. Clin Oral Implants Res 2003;14:720-6.

8. Cune M, Burgers M, van Kampen F, de Putter C, van der Bilt A. Mandibular overdentures retained by two implants: 10-year results from a crossover clinical trial comparing ball-socket and bar-clip attachments. Int J Prosthodont 2010;23:310-7.

9. Kularashmi BS, Anand MV, Bettie NF, Ramachandiran H. A telescopic retainer prosthesis in full mouth rehabilitation. J Pharm Bioallied Sci 2015;7 Suppl 2:S804-5.

10. Bayer S, Stark H, Gölz L, Keilig L, Kraus D, Hansen A, et al. Telescopic crowns: Extra-oral and intra-oral retention force measurement - in vitro/ in vivo correlation. Gerodontology 2012;29:e340-7.

11. Dittmann B, Rammelsberg P. Survival of abutment teeth used for telescopic abutment retainers in removable partial dentures. Int J Prosthodont 2008;21:319-21.

12. Kern JS, Kern T, Wolfart S, Heussen N A systematic review and meta-analysis of removable and fixed implant-supported prostheses in edentulous jaws: Post-loading implant loss. Clin Oral Implants Res 2016;27:174-95.

13. Carr AB. Successful long-term treatment outcomes in the field of osseointegrated implants: Prosthodontic determinants. Int J Prosthodont 1998;11:502-12.

14. Guckes AD, Scurria MS, Shugars DA. A conceptual framework for understanding outcomes of oral implant therapy. J Prosthet Dent 1996;75:633-9.

15. Berglundh T, Persson L, Klinge B. A systematic review of the incidence of biological and technical complications in implant dentistry reported in prospective longitudinal studies of at least 5 years. J Clin Periodontol 2002;29 Suppl 3:197-212.

16. Papaspyridakos P, Chen CJ, Chuang SK, Weber HP, Gallucci GO. A systematic review of biologic and technical complications with fixed implant rehabilitations for edentulous patients. Int J Oral Maxillofac Implants 2012;27:102-10.

17. Moher D, Liberati A, Tetzlaff J, Altman DG; PRISMA Group. Preferred reporting items for systematic reviews and meta-analyses: The PRISMA statement. Int J Surg 2010;8:336-41.

18. Center For Evidence Based Medicine. Askig Focused Questions. Oxford: University of Oxford; 2014. Available from: http://www.cebm. net. [Last accessed on 2014 Jan 01].

19. Higgins JP, Altman DG, Gøtzsche PC, Jüni P, Moher D, Oxman AD, et al. The Cochrane Collaboration's tool for assessing risk of bias in randomised trials. BMJ 2011;343:d5928.

20. Review Manager (RevMan) [Computer program]. Version 5.3. Copenhagen: The Nordic Cochrane Centre, the Cochrane Collaboration, 2014.

21. Harris RJ, Bradburn MJ, Deeks JJ, Harbord RM, Altman DG, Sterne JA. Metan: Fixed-and random-effects meta-analysis. Stata J 2008;8:3-28.

22. Cepa S, Koller B, Spies BC, Stampf S, Kohal RJ. Implant-retained prostheses: Ball vs. conus attachments - A randomized controlled clinical trial. Clin Oral Implants Res 2017;28:177-185.

23. Eitner S, Schlegel A, Emeka N, Holst S, Will J, Hamel J. Comparing bar and double-crown attachments in implant-retained prosthetic reconstruction: A follow-up investigation. Clin Oral Implants Res 2008;19:530-7.

24. Elsyad MA, Khairallah AS. Chewing efficiency and maximum bite force with different attachment systems of implant overdentures: A crossover study. Clin Oral Implants Res 2017;28:677-82.

25. Heuer W, Kettenring A, Demling A, Stumpp SN, Gellermann E, Winkel A, et al. Microbial diversity of peri-implant biofilms on implant 
fixed bar and telescopic double crown attachments. J Oral Implantol 2013;39:648-54.

26. Khalid T, Yunus N, Ibrahim N, Elkezza A, Masood M. Patient-reported outcome and its association with attachment type and bone volume in mandibular implant overdenture. Clin Oral Implants Res 2017;28:535-42.

27. Krennmair G, Seemann R, Weinländer M, Piehslinger E. Comparison of ball and telescopic crown attachments in implant-retained mandibular overdentures: A 5-year prospective study. Int J Oral Maxillofac Implants 2011;26:598-606.

28. Krennmair G, Sütö D, Seemann R, Piehslinger E. Removable four implant-supported mandibular overdentures rigidly retained with telescopic crowns or milled bars: A 3-year prospective study. Clin Oral Implants Res 2012;23:481-8.

29. Krennmair G, Weinländer M, Krainhöfner M, Piehslinger E. Implant-supported mandibular overdentures retained with ball or telescopic crown attachments: A 3-year prospective study. Int J Prosthodont 2006;19:164-70.

30. Cicciu M, Bramanti E, Matacena G, Guglielmino E, Risitano G. FEM evaluation of cemented-retained versus screw-retained dental implant single-tooth crown prosthesis. Int J Clin Exp Med 2014;7:817-25.

31. Lauritano F, Runci M, Cervino G, Fiorillo L, Bramanti E, Cicciù M. Three-dimensional evaluation of different prosthesis retention systems using finite element analysis and the Von Mises stress test. Minerva Stomatol 2016;65:353-67.

32. Meriç G, Erkmen E, KurtA, Eser A, Ozden AU. Biomechanical comparison of two different collar structured implants supporting 3-unit fixed partial denture: A 3-D FEM study. Acta Odontol Scand 2012;70:61-71.
33. Glenny AM, Nieri M, Worthington $\mathrm{H}$, Espostio M. The importance of the study design: From the case report to the randomised controlled clinical trial. Eur J Oral Implantol 2008;1:317-21.

34. Sylvester RJ, Canfield SE, Lam TB, Marconi L, MacLennan S, Yuan Y, et al. Conflict of evidence: Resolving discrepancies when findings from randomized controlled trials and meta-analyses disagree. Eur Urol 2017;71:811-9.

35. MacEntee MI, Walton JN, Glick N. A clinical trial of patient satisfaction and prosthodontic needs with ball and bar attachments for implant-retained complete overdentures: Three-year results. J Prosthet Dent 2005;93:28-37.

36. Watson GK, Payne AG, Purton DG, Thomson WM. Mandibular overdentures: Comparative evaluation of prosthodontic maintenance of three different implant systems during the first year of service. Int J Prosthodont 2002;15:259-66.

37. Karabuda C, Yaltirik M, Bayraktar M. A clinical comparison of prosthetic complications of implant-supported overdentures with different attachment systems. Implant Dent 2008;17:74-81.

38. Hartman GA, Cochran DL. Initial implant position determines the magnitude of crestal bone remodeling. J Periodontol 2004;75:572-7.

39. Hermann JS, Buser D, Schenk RK, Schoolfield JD, Cochran DL. Biologic Width around one- and two-piece titanium implants. Clin Oral Implants Res 2001;12:559-71.

40. Naert I, Alsaadi G, van Steenberghe D, Quirynen M. A 10-year randomized clinical trial on the influence of splinted and unsplinted oral implants retaining mandibular overdentures: Peri-implant outcome. Int J Oral Maxillofac Implants 2004;19:695-702. 